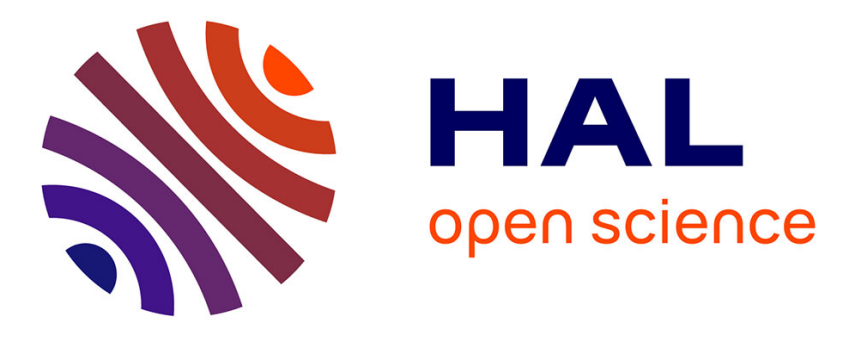

\title{
Qu'est-ce que l'intégration? Analyse de discours de formateurs et formatrices linguistiques intervenant auprès de migrants adultes en France
}

\author{
Maude Vadot
}

\section{- To cite this version:}

Maude Vadot. Qu'est-ce que l'intégration? Analyse de discours de formateurs et formatrices linguistiques intervenant auprès de migrants adultes en France. J.-C. Beacco, H.-J. Krumm, D. Little, \& P. Thalgott. L'intégration linguistique des migrants adultes: les enseignements de la recherche, Mouton De Gruyter (en collaboration avec le Conseil de l'Europe), 2017, 9783110477498. 10.1515/9783110477498-053 . halshs-01500576

\section{HAL Id: halshs-01500576 \\ https://shs.hal.science/halshs-01500576}

Submitted on 3 Apr 2017

HAL is a multi-disciplinary open access archive for the deposit and dissemination of scientific research documents, whether they are published or not. The documents may come from teaching and research institutions in France or abroad, or from public or private research centers.
L'archive ouverte pluridisciplinaire HAL, est destinée au dépôt et à la diffusion de documents scientifiques de niveau recherche, publiés ou non, émanant des établissements d'enseignement et de recherche français ou étrangers, des laboratoires publics ou privés.

\section{(ㅇ)(1) $\$$}

Distributed under a Creative Commons Attribution - NonCommercial - NoDerivatives $\mid 4.0$ 


\section{Qu'est-ce que l'intégration?}

\section{Analyse de discours de formateurs et formatrices linguistiques intervenant auprès de migrants adultes en France}

Résumé : L'institutionnalisation de la formation linguistique des migrants adultes en France s'est accompagnée du choix du terme intégration, consacré par la parution du Référentiel Français Langue d'Intégration fin 2011. On analyse ici comment des formateurs spécialistes du domaine actualisent et commentent les lexèmes intégration, intégrer, s'intégrer et être intégré. On montre ainsi que si une partie d'entre eux est consciente de la mémoire discursive charriée par le terme, le concept reste pour eux difficilement saisissable. Ces résultats permettent d'envisager quelques orientations pour la formation initiale et continue des formateurs linguistiques.

Abstract: The institutionalisation of language education for adult migrants in France has gone hand in hand with the choice of the term intégration, as laid down in the Référentiel Français Langue d'Intégration [French as a Language of Integration - Reference guide] published at the end of 2011. This article analyses how specialist trainers in this field are actualizing and reacting to the lexemes intégration, intégrer, s'intégrer and être intégré. It shows that, while some of them are aware of the term's discursive load, the concept remains difficult for them to grasp. These results could give rise to guidelines on the initial and further training of language trainers.

\section{Introduction}

La parution du Référentiel Français Langue d'Intégration (FLI) (Vicher et al. 2011), sur commande de la Direction de l'Accueil, de l'Intégration et de la Citoyenneté, a été suivie de vives réactions - circonscrites au domaine de formation et de recherche concerné - portant, entre autres, sur le recours au terme intégration. Cette publication réactivait en effet la polémique touchant à l'emploi et la prescription des termes constituant le paradigme insertion, intégration, assimilation - et plus récemment inclusion et acculturation (Vadot 2016). Ces termes,

Maude Vadot, Laboratoire Dipralang EA739, Université Paul-Valéry Montpellier 3, E-mail : maude_vadot@yahoo.fr

DOI 10.1515/9783110477498-053, (cc) BY-NC-ND (c) 2017 Maude Vadot, published by De Gruyter. This work is licensed under the Creative Commons Attribution-NonCommercial-NoDerivs 3.0 License. 
qui ont circulé d'un camp politique à un autre dans les dernières décennies, sont souvent critiqués comme manquant de contenu sémantique : il est de fait assez rare d'en trouver des définitions qui permettent de les mettre en contraste. Pour autant, les changements de mots accompagnent parfois des changements idéologiques qui ont, pour leur part, des conséquences en matière d'action publique et de travail social (Lemière 2006).

Je m’intéresse ici aux actualisations réalisées par des formateurs et formatrices intervenant auprès de migrants adultes en France, pour dégager au moins partiellement la mise en discours et en sens de la dénomination intégration ainsi effectuée. L'enjeu consiste à approcher la façon dont ils se saisissent des enjeux des formations linguistiques à visée d'insertion ou d'intégration. Comment conçoivent-ils l'intégration des personnes migrantes ? Sont-ils conscients des querelles de mots et si oui, quel sens prennent-elles pour eux ? Comment se situent-ils dans ces débats?

\section{Méthodologie de recueil et d'analyse}

Le corpus est composé d'entretiens semi-directifs menés auprès de trois formateurs et quatorze formatrices âgés de 25 à 45 ans, en poste ou en recherche d'emploi au moment de l'entretien. Tous ont entre un et dix ans d'expérience auprès de migrants, principalement en organisme de formation. Le protocole suivi consistait à aborder les thèmes d'entretien sans prononcer aucun des termes du paradigme, afin de ne pas imposer l'emploi de l'un ou de l'autre. Ce n'est que lorsqu'aucun terme n'avait été prononcé lors des premiers échanges qu'on confrontait l'interlocuteur au lexème intégration, le plus souvent en l'interrogeant sur sa connaissance et sa réception du Référentiel FLI, et ce pour observer sa réaction sans pour autant initier la constitution du terme en objet de discours.

Afin de mettre au jour les éléments du programme de sens actualisés par les formateurs et formatrices employant les unités lexicales intégration, intégrer, s'intégrer et être intégré, je me suis inspirée des propositions de l'Analyse de Discours à Entrée Lexicale (Née \& Veniard 2012), et notamment d'une sémantique des constructions. Il s'agit de relever toutes les occurrences des unités étudiées et d'observer non seulement la fréquence à laquelle les locuteurs y ont recours, mais également leur mise en syntagme au sein des constructions nominales et des constructions verbales dans lesquelles elles apparaissent, puis d'en dégager les effets. J'ai exclu du relevé les occurrences incluses dans les expressions " contrat d'accueil et d'intégration », " français langue d'intégration » et « ouvrir l'école aux parents pour réussir l'intégration », syntagmes 
quasi-lexicalisés appartenant au discours institutionnel et repris dialogiquement par les formateurs.

\section{Posture critique vs. acceptation du lexème}

Le premier résultat observable consiste en ce qu'on peut diviser les formateurs en deux groupes. Neuf d'entre eux ne se positionnent pas face au terme et, pour la plupart, l'emploient sans difficulté. Incarnant une posture différente, les huit autres commentent le lexème de façon critique, voire affirment refuser d'y avoir recours au quotidien :

(1) parler des valeurs enfin ou même dire le mot intégration ${ }^{1}$ ça me donne de l'urticaire c'est <((en riant légèrement)) pas possible> $\left(\right.$ Alice $\left.^{2}\right)$

Faisant du mot l'objet du discours, les formateurs du second groupe ont tous recours à des actualisations autonymiques du lexème nominal ou verbal, dans lesquelles le signe renvoie à lui-même en tant que signe. Les commentaires énoncés relèvent témoignent de la réflexivité des locuteurs sur la langue et les discours, qui est mise au service de l'argumentation (Micheli 2011 ; Sitri 2003) : les locuteurs visent alors à problématiser l'emploi du terme.

Pour leur part, les formateurs qui ne critiquent pas le terme ne réalisent pas, à une exception près, d'emplois autonymiques des lexèmes. La seule qui le fait vise, à l'inverse des formateurs critiques, à valider le recours au terme :

(2) intégration oui il faut que: enfin ça par contre euh / je le: / je lui mets pas de connotation derrière (Aurélie)

Ces emplois autonymiques ne seront pas pris en compte dans les analyses qui vont suivre dans la mesure où ils constituent des mises à distance du terme par les formateurs concernés. Il en va de même pour les énoncés appartenant au discours rapporté, ainsi que pour les actualisations faisant l'objet d'une modalisation autonymique, qui témoignent également d'un refus au moins partiel de la responsabilité énonciative par le locuteur.

Cependant, malgré les critiques qu'ils formulent et le rejet parfois virulent qu'ils expriment, six des huit formateurs critiques ont recours au terme en emploi standard sans qu'il leur soit imposé.

1 C'est moi qui souligne.

2 Tous les prénoms ont été modifiés pour garantir l'anonymat des participants. 


\section{Se saisir du concept. Caractéristiques syntagmatiques des actualisations de la dénomination intégration et de ses variantes lexématiques}

\subsection{Recours au nom vs. recours aux verbes}

Les caractéristiques des actualisations réalisées par les deux groupes de formateurs, au total 144 occurrences, sont assez proches. Un élément distingue toutefois les deux profils : les formateurs critiques ont plus fréquemment recours aux formes verbales (69\%) qu'à la forme nominale du lemme (31\%), là où la tendance est inverse pour les formateurs non critiques (40 \% vs. 60 \%). On peut faire l'hypothèse que critiquant le nom et faute d'avoir recours à une autre dénomination, les formateurs critiques sont obligés d'actualiser les formes verbales du lemme.

Or le recours au nom intégration permet une abstraction plus grande. Là où il est en effet possible de dire :

(3) des contenus qui sont tournés vers l'intégration (Étienne)

sans préciser d'arguments (actants et actés), l'emploi d'une forme verbale du lemme oblige le locuteur à préciser au moins un argument dans le cas de la construction passive ou réfléchie, voire deux en construction transitive active :

(4) elles sont pas du tout intégrées (Étienne)

(5) on va essayer de t'intégrer (Lydie)

Par ailleurs, le nom permet de ne pas se prononcer quant à l'agentivité ou à l'inscription du procès dans le temps. Le syntagme "l'intégration des migrants dans la société » pourrait ainsi être reformulé en :

- les migrants s'intègrent dans la société

- on a intégré les migrants dans la société

- les migrants seront intégrés dans la société

- etc.

Le choix énonciatif effectué de façon préférentielle par les formateurs non critiques est donc générateur d'énoncés dont le degré d'abstraction est plus élevé. 


\subsection{Actualisations nominales}

La majorité des occurrences (65\%) se présente sous la forme « article défini + nom ». Les occurrences faisant intervenir d'autres déterminants sont très rares (3 au total) ; elles produisent un effet de sens bien différent qui laisse envisager la possibilité d'intégrations différenciées selon les personnes, ou d'une intégration par pallier, comme le laissent entendre les exemples suivants :

(6) une intégration de base (Laura)

(7) mon intégration à moi (Sylvie)

Par ailleurs, le syntagme nominal est très souvent réduit à sa structure minimale : seuls $20 \%$ des syntagmes nominaux comportent une épithète ou un complément du nom. Il en résulte une très faible restriction du sens du lexème. De plus, ces restrictions du sens sont peu spécifiques : deux des trois arguments relevés réfèrent à l'énonciateur, qui parle alors de son expérience en tant qu'enseignant de français à l'étranger. Le dernier désigne une catégorie générique (« des personnes »). Pour leur part, les circonstants actualisés, indiquant des lieux, sont situés à une échelle très large, celle du pays ou de la société. À une exception près, aucun locuteur ne qualifie de domaine dans ou par lequel le processus d'intégration se réalise.

\subsection{Actualisations verbales}

Un premier constat concerne la répartition des occurrences entre les trois formes verbales du lemme : sur la totalité des emplois, la préférence est largement donnée aux constructions en être intégré (51\%) et en s'intégrer (43\%). De plus, les constructions au passif ne reçoivent jamais de complément d'agent qui viendrait préciser l'actant du procès mis en scène : pour être intégré comme pour s'intégrer, le seul argument exprimé est le sujet de la forme verbale. Il en résulte que dans l'ordre du discours des formateurs, la responsabilité de l'intégration est placée entre les mains de ceux qui en sont l'objet - ici les adultes migrants.

L'observation des arguments des formes verbales conjuguées permet de constater que dans les deux tiers des cas, les formateurs mettent en scène des catégories génériques (« la personne », « les migrants »), évoquant ainsi des cas virtuels. Seuls un tiers des énoncés réfèrent à des situations vécues - la leur ou celle d'un tiers.

Enfin, un tiers des occurrences sont dotées de compléments entrant dans la construction des syntagmes verbaux relevés, qui se répartissent entre spécification d'un lieu ou d'un espace social dans lequel l'intégration a lieu, des 
moyens par lesquels elle est réalisée, ou encore d'un degré d'intensité. Les dénominations employées, qu'il s'agisse de lieux ou de moyens envisagés, restent là aussi très générales. Enfin, il est intéressant de constater que la moitié de ces compléments a trait à l'intensité du procès s'intégrer ou de l'état être intégré.

\section{Conclusion}

A l'issue de ces analyses, on peut avancer que la notion d'intégration est, dans les discours, appréhendée de façon très globalisante et vague par les formateurs et formatrices. Les formateurs parlent rarement de situations particulières qu'ils seraient en mesure de comparer les unes aux autres pour illustrer ou étayer leur propos ; les lieux ou les groupes humains mobilisés restent très vastes et généraux. Les processus sont donc appréhendés d'une façon tellement globale qu'il n'est pas possible d'en saisir les tenants et les aboutissants, les modalités, les spécificités, les variables, etc. De plus le rôle de la société d'accueil, de ses institutions, de ses membres, de son histoire, etc. est totalement " invisibilisé ». Cela revient alors à désigner les migrants comme seuls acteurs du processus, ce qui ouvre ensuite la possibilité de les renvoyer à leurs prétendues seules responsabilités.

Bien sûr, l'étude des constructions syntaxiques ne dit pas tout, et une analyse thématique des entretiens permet de faire apparaître des voies et des domaines que les formateurs identifient comme vecteurs d'intégration. Il reste qu'en discours, et ce malgré la formulation d'éventuelles critiques, l'intégration constitue une réalité dont les modalités et les enjeux restent à travailler.

Dans un moment où, en France, la formation linguistique des migrants est de plus en plus liée à des orientations idéologiques, et où le rapport entre langue et intégration semble aller de soi, la construction de la professionnalité des formateurs passe également par la construction de connaissances sociologiques sur les processus d'intégration et la manière dont ils ont été travaillés par le politique au cours des dernières décennies. Ces éléments font partie intégrante de la « contextualisation du terrain », comme l'a souligné Bretegnier en s'attachant à décrire les compétences et les ressources nécessaires à la construction d'une posture de réflexivité éthique (Bretegnier 2011). Il semble donc nécessaire de réfléchir aux manières dont on peut améliorer dans ce sens les formations de formateurs, tant initiales que continues. 


\section{Références}

Authier-Revuz, Jacqueline. 1995. Ces mots qui ne vont pas de soi. Boucles réflexives et non coïncidences du dire. Paris : Larousse.

Bretegnier, Aude. 2011. Formation linguistique en contextes d'insertion : vers un cadre de référence(s) en matière de professionnalité de formateurs. In Aude Bretegnier (dir.), Formation linguistique en contextes d'insertion : compétences, posture, professionnalité, concevoir un cadre de référence(s), 213-242. Berne, Berlin \& Bruxelles : Peter Lang.

Lemière, Jacques. 2006. De l'importance et de l'urgence d'étendre dans le travail social, la critique des catégories d'intégration et d'immigré. In Élisabeth Prieur, Emmanuel Jovelin \& Martine Blanc (dir.). Travail social et immigration, 91-103. Paris : L'Harmattan.

Micheli, Raphaël. 2011. Quand l'affrontement porte sur les mots en tant que mots : polémique et réflexivité langagière. Semen. Revue de sémio-linguistique des textes et discours 31. 97-112.

Née, Émilie \& Marie Veniard. 2012. Analyse du Discours à Entrée Lexicale (A.D.E.L.) : le renouveau par la sémantique ?. Langage et société 140. 15-28.

Sitri, Frédérique. 2003. L'autonymie dans la construction des objets de discours. In Jacqueline Authier-Revuz, Marianne Doury \& Sandrine Reboul-Touré (dir.). Parler des mots. Le fait autonymique en discours, 205-216. Paris : Presses Sorbonne Nouvelle.

Vadot, Maude. 2016. De quoi INTÉGRATION est-il le nom ? L'importation d'une querelle de mots dans le champ de la formation linguistique des migrants. Argumentation et Analyse du Discours 17. http://aad.revues.org/2228 (consulté le 14 juillet 2015).

Vicher, Anne, Hervé Adami, Amandine Bergère, Sophie Étienne, Pascal Lambert, Gaëlle Poirrier \& Claire Verdier. 2011. Référentiel FLI - Français Langue d'Intégration. Direction de l'Accueil, de l'Intégration et de la Citoyenneté. http://www.immigration.interieur.gouv. $\mathrm{fr} /$ content/download/38544/292981/file/FLI-Referentiel.pdf. (consulté le 02/12/2016). 
